\title{
Calcific Tendinopathy of the Gluteus Medius Mimicking Lumbar Radicular Pain Successfully Treated With Barbotage: A Case Report
}

\author{
Hannae Jo, MD, Gowun Kim, MD, Sora Baek, MD, PhD, Hee-won Park, MD
}

Department of Rehabilitation Medicine, Kangwon National University Hospital, Chuncheon, Korea

We report a case of calcific tendinopathy of the gluteus medius initially misdiagnosed as a lumbar herniated intervertebral disc. It was successfully treated with barbotage under ultrasonographic guidance finally. A 56-yearold woman was referred to interventional pain clinic for right hip pain due to an L5-S1 disc herniation. Serial L5 and S1 spinal nerve root blocks and epidural steroid injections were administered. However, pain relief was sustained only for a very short period. Plain radiography of the right hip revealed a solid calcific nodule at adjacent to the insertion site of the gluteus medius tendon. Physical modalities and extracorporeal shock wave therapy failed to improve the pain. Therefore, we attempted ultrasound-guided barbotage of the calcification. Barbotage was performed twice serially and her pain was considerably improved. At 6-month follow-up, the calcification was completely resolved.

Keywords Gluteus medius, Calcific tendinopathy, Barbotage

\section{INTRODUCTION}

Calcific tendinopathy presents calcific deposits in specific tendons. It is also known as calcifying tendinitis, periarticular apatite deposition disease, or calcifying periarticulitis [1]. The rotator cuff tendon is the most commonly affected site of calcific tendinopathy. However, Achilles tendon and quadriceps tendon are also fre-

Received June 1, 2015; Accepted August 25, 2015

Corresponding author: Hee-won Park

Department of Rehabilitation Medicine, Kangwon National University Hospital, 156 Baengnyeong-ro, Chuncheon 24289, Korea

Tel: +82-33-248-7703, Fax: +82-33-258-9097, E-mail: hwp9980@gmail. com

(c) This is an open-access article distributed under the terms of the Creative Commons Attribution Non-Commercial License (http://creativecommons. org/licenses/by-nc/4.0) which permits unrestricted noncommercial use, distribution, and reproduction in any medium, provided the original work is properly cited.

Copyright (C) 2016 by Korean Academy of Rehabilitation Medicine quent sites of calcific tendinopathy. Calcific tendinopathy of the gluteus medius has been sporadically reported. It usually causes pain in the lateral aspect of the hip which may simulate lumbar radiculopathy [2-5]. Here we report a case of calcific tendinopathy in the gluteus medius tendon initially misdiagnosed as lumbar radiculopathy but successfully treated with needling, lavage, and aspiration (barbotage) under ultrasound guidance. To the best of our knowledge, there is the first report of calcific tendinopathy in the gluteus medius tendon treated with barbotage.

\section{CASE REPORT}

A 56-year-old woman with pain in the right lateral hip area was referred for a $\mathrm{C}$-arm guided epidural steroid injection. She had previously sustained a L5 compres- 
sion fracture after a traffic accident which was treated conservatively for 3 months (Fig. 1A). After 3 months of conservative treatment, she had partial resolution of back pain. However, she complained of new pain in the right lateral hip area. The pain was not localized in specific area. It was radiated to lateral thigh. Straight leg raising test was positive. Magnetic resonance imaging (MRI) of the lumbar spine performed 4 months after the traffic accident revealed an L5-S1 disc herniation (Fig. 1B). L5 and S1 root blocks with epidural steroid injections were performed three times for the radicular pain suspected to be from the L5-S1 disc herniation.

Despite injection, the pain relief was sustained only for a very short period. After administration of the first L5 epidural steroid injection, the pain was decreased and maintained for 3 to 4 days. However, the second L5 epidural steroid injection was ineffective. S1 epidural steroid injection was administered as the last try. It had no noticeable result. The dose of the corticosteroid was $40 \mathrm{mg}$ for the first injection and $20 \mathrm{mg}$ for the second and the third injections. A detailed assessment of history and physical examination were performed for further evaluation. The patient complained of a clicking sensation while walking. Deep palpation over the right greater trochanter reproduced the pain. Laboratory studies showed normal levels of serum alkaline phosphatase, erythrocyte sedimentation rate, and C-reactive protein. Plain radiographs and an ultrasound of the right hip revealed a 2.0 $\mathrm{cm} \times 0.7 \mathrm{~cm}$ sized solid calcific nodule adjacent to the insertion site of the gluteus medius tendon between the greater trochanter and iliotibial band (Fig. 2A, 2B). The patient was diagnosed as having calcific tendinopathy of the gluteus medius tendon. She underwent physical therapy including hot packs and ultrasound for 1 week. After physical therapy, ultrasonography-guided intrabursal injection of triamcinolone (20 mg) was done. However,
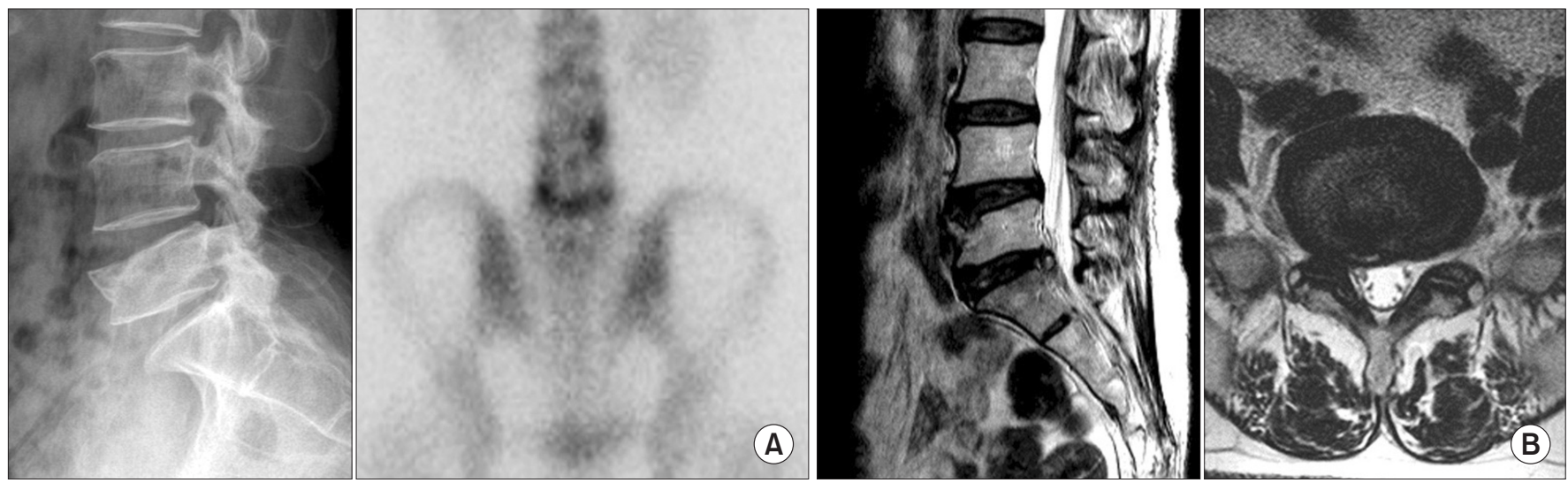

Fig. 1. (A) Simple X-ray and bone scan finding of L5 compression fracture. (B) Sagittal and axial T2-weighted magnetic resonance images showing L5-S1 intervertebral disc herniation.
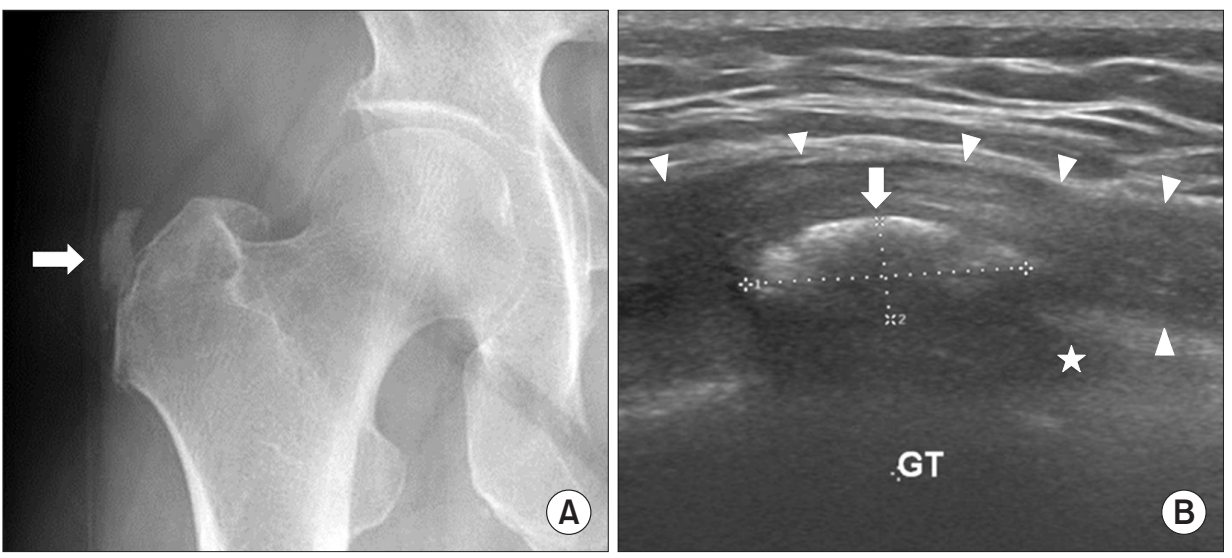

Fig. 2. (A) An anteroposterior radiograph of the right hip showing an amorphous calcification (white arrow) adjacent to the greater trochanter. (B) Ultrasonography of the right hip showing a solid calcific nodule (white arrow) at $2.0 \mathrm{~cm} \times 0.7 \mathrm{~cm}$ in size adjacent to the insertion site of the gluteus medius tendon (asterisk) between the greater trochanter (GT) and the iliotibial band (arrowheads). 
both treatments showed little effect for the relief of pain. It seemed that the pain was induced by mechanical irritation of calcific nodule. Therefore, we made a decision to remove calcific nodule. The first choice for the disintegration of calcific nodule was extracorporeal shock wave therapy (ESWT). A radial-type ESWT device (Gymna ShockMaster 500; GymnaUniphy, Bilzen, Belgium) was used. In each session the patient received 2,000 pulses of shockwave by the intensity of $0.16 \mathrm{~mJ} / \mathrm{mm}^{2}$. The diameter of an applicator was $15 \mathrm{~mm}$. Total three sessions (twice per week) of treatment were done and discontinued due to aggravation of pain. After failure of ESWT, ultrasonography-guided barbotage was planned. The procedure was performed using a one-needle technique. The transducer was approached with an in-plane view. Local anesthesia ( $1 \%$ lidocaine) was administered prior to barbotage. The calcification was punctured using a 20-gauge needle. Saline $(0.9 \%)$ was flushed with a $15-\mathrm{mL}$ syringe into the fragmented calcific deposit. Liquefied calcium crystal was aspirated (Fig. 3). Once the syringe was filled with calcium deposit micro-crystals, no saline was aspirated. A new syringe filled with saline was then used. After using approximately $100 \mathrm{~mL}$ of $0.9 \%$ saline used for the lavage, a substantial amount of calcium deposit was washed out and the needle was withdrawn.

After performing barbotage twice, more than half of the calcific deposit was aspirated successfully (Fig. 4A, 4B). Complete resolution of hip pain was achieved. The first barbotage brought instant and considerable pain relief. The second barbotage did not bring noticeable effect. Considering the dose of triamcinolone used for epidural injections, additional bursal steroid injection was not performed. The patient did not complained of pain aggravation. The clicking sensation while walking was resolved. She felt much more comfortable with her gait. Follow-up radiography at 6 months (Fig. 5) showed
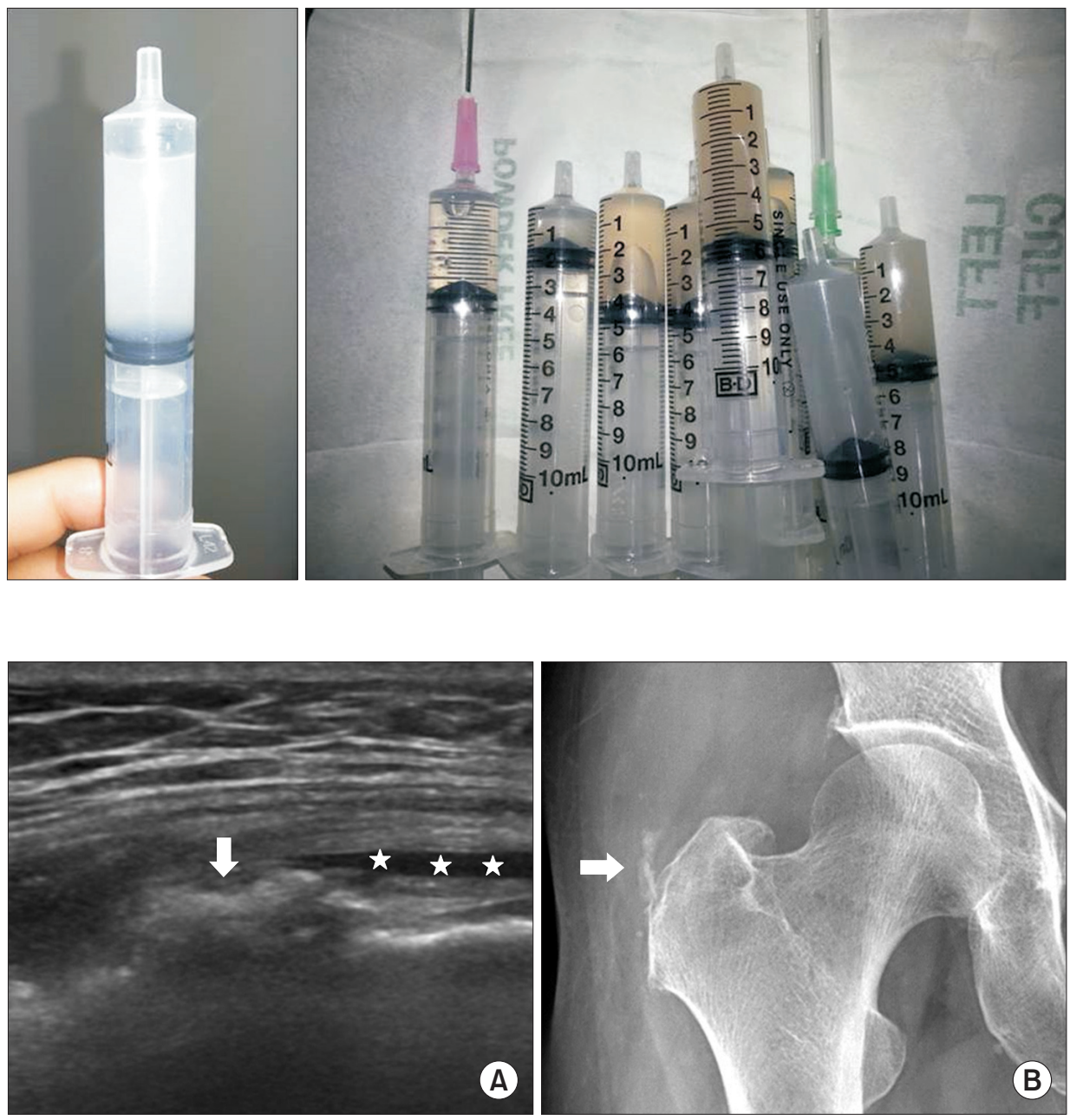

Fig. 3. Aspirated calcium deposit in $15 \mathrm{~mL}$ syringe.

Fig. 4. (A) Ultrasonography of the same site immediately after ultrasonography-guided barbotage showing significant reduction in the size of the calcific deposit (white arrow). A fluid collection (asterisk) of saline remained after the procedure. (B) The size of the calcification was reduced significantly after barbotage (white arrow). 


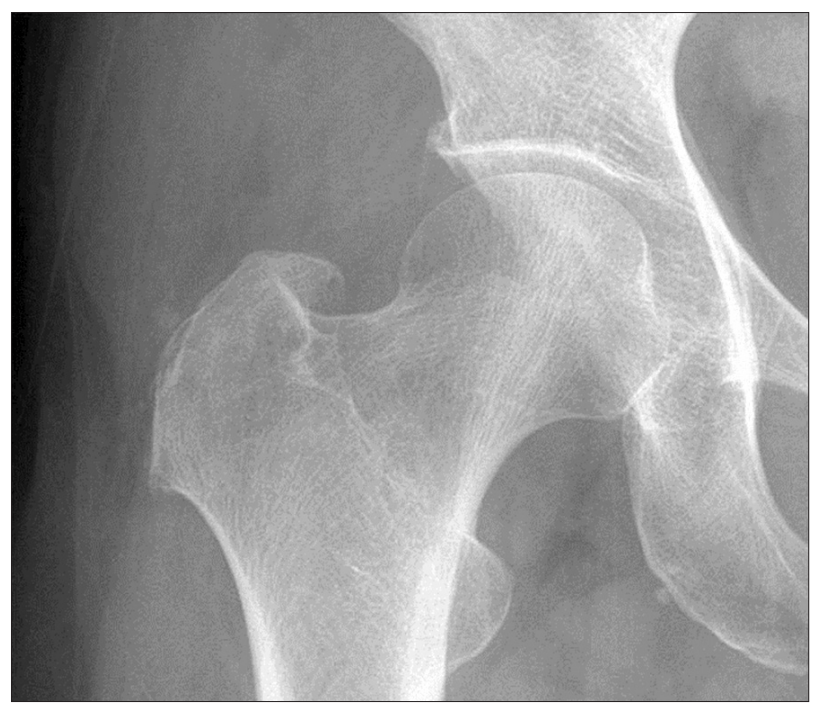

Fig. 5. Anteroposterior radiograph of the right hip at 6 -month follow-up showing near complete resolution of the calcific deposit.

near-complete resolution of calcific deposits. The patient remained pain free.

\section{DISCUSSION}

Few cases of calcific tendinopathy in the gluteus medius tendon have been reported [2-8]. Calcific tendinopathy in the gluteus medius tendon presenting as lateral hip pain may be misdiagnosed as lumbar radiculopathy. Because the iliotibial tract and the lumbar dermatomes overlap anatomically, symptoms of calcific tendinopathy in the gluteus medius tendon may mimic lumbar radiculopathy symptoms. Awareness of both entities and a detailed history and physical examination can help differentiate the two diseases. Pain originating from the greater trochanter does not extend distal to the proximal tibia, whereas pain originating from true nerve root irritation extends into the lower leg and foot. In addition, patients with lumbar radiculopathy do not have reproduction of pain with pressure over the greater trochanter [9]. Calcific tendinopathy is usually easily diagnosed based on clinical suspicion and typical radiographic or ultrasonographic findings. Additional computed tomography or MRI can help in some instances [2,5,7]. High-resolution ultrasonography has great potential in investigating the morphology of calcified deposits.

Calcific tendinopathy is usually a self-limiting condi- tion. Most patients can be treated with conservative treatments including non-steroidal anti-inflammatory drugs and physiotherapy. When these treatments fail, minimally invasive treatments such as ESWT and barbotage as in this case are indicated before surgery. We tried radial-type ESWT. However, calcification was not eliminated. In contrast with focused-type ESWT, radial-type ESWT generate more spreading shockwaves. It is difficult to ensure proper delivery of energy at the proper strength when the target location is deep as in our case. Barbotage is a medical procedure that involves repeated injection and aspiration. Ultrasonography-guided barbotage has proved to be effective in calcific tendinopathy of the rotator cuff tendon $[9,10]$.

Different modalities have been suggested for calcific tendinopathy in the gluteus medius. Conservative management such as non-steroidal anti-inflammatory drugs has shown favorable outcomes [2,6,7]. Arthroscopic excision of calcific debris has been used for intractable cases [6]. Unusual treatment methods such as an endoscopic approach, acupuncture, and small needle scalpel therapy have been successfully used to treat calcific tendinopathy of the gluteus medius $[3,4]$.

The natural course of calcific tendinopathy in the gluteus medius treated by barbotage is not well documented. To our knowledge, this is the first report of calcific tendinopathy in the gluteus medius treated by barbotage. In rotator cuff tendon calcification, post-procedure pain due to bursitis may occur. Post-procedure intrabursal steroid injection is recommended. However, we did not inject steroids after barbotage because considerable doses of steroids had been administered during the previous epidural procedures without improving pain. Barbotage can be performed using a one-needle or two-needle technique, with the two-needle technique being possibly more effective. In this gluteus medius tendon case, with its deep location and the surrounding tissues such as the iliotibial tract and fat, the two-needle technique was considered to be difficult. The one-needle technique absorbed the calcific deposit successfully.

In conclusion, calcific tendinopathy of the gluteus medius should be considered as a differential diagnosis in patients presenting with lateral hip pain. Ultrasonography-guided barbotage was effective in managing the pain. The calcification was successfully resolved after treatment. 


\section{CONFLICT OF INTEREST}

No potential conflict of interest relevant to this article was reported.

\section{REFERENCES}

1. Oliva F, Via AG, Maffulli N. Calcific tendinopathy of the rotator cuff tendons. Sports Med Arthrosc 2011; 19:237-43.

2. Paik NC. Acute calcific tendinitis of the gluteus medius: an uncommon source for back, buttock, and thigh pain. Semin Arthritis Rheum 2014;43:824-9.

3. Kandemir U, Bharam S, Philippon MJ, Fu FH. Endoscopic treatment of calcific tendinitis of gluteus medius and minimus. Arthroscopy 2003;19:E4.

4. Lin W, Liu CY, Tang CL, Hsu CH. Acupuncture and small needle scalpel therapy in the treatment of calcifying tendonitis of the gluteus medius: a case report. Acupunct Med 2012;30:142-3.

5. Yang I, Hayes CW, Biermann JS. Calcific tendinitis of the gluteus medius tendon with bone marrow edema mimicking metastatic disease. Skeletal Radiol 2002;31:359-61.

6. Park SM, Baek JH, Ko YB, Lee HJ, Park KJ, Ha YC. Management of acute calcific tendinitis around the hip joint. Am J Sports Med 2014;42:2659-65.

7. Sakai T, Shimaoka Y, Sugimoto M, Koizumi T. Acute calcific tendinitis of the gluteus medius: a case report with serial magnetic resonance imaging findings. J Orthop Sci 2004;9:404-7.

8. Callaghan BD. Unusual calcification in the region of the gluteus medius and minimus muscles. Australas Radiol 1977;21:362-6.

9. del Cura JL, Torre I, Zabala R, Legorburu A. Sonographically guided percutaneous needle lavage in calcific tendinitis of the shoulder: short- and long-term results. AJR Am J Roentgenol 2007;189:W128-34.

10. Serafini G, Sconfienza LM, Lacelli F, Silvestri E, Aliprandi A, Sardanelli F. Rotator cuff calcific tendonitis: short-term and 10-year outcomes after two-needle us-guided percutaneous treatment: nonrandomized controlled trial. Radiology 2009;252:157-64. 